\title{
Moment Aberration Projection for Nonregular Fractional Factorial Designs
}

\author{
Hongquan $\mathrm{XU}$ \\ Department of Statistics \\ University of California \\ Los Angeles, CA 90095 \\ (hqxu@stat.ucla.edu)
}

\author{
Lih-Yuan DENG \\ Department of Mathematical Sciences \\ The University of Memphis \\ Memphis, TN 38152 \\ (lihdeng@memphis.edu)
}

\begin{abstract}
Nonregular fractional factorial designs, such as Plackett-Burman designs, are widely used in industrial experiments for run size economy and flexibility. A novel criterion, called moment aberration projection, is proposed to rank and classify nonregular designs. It measures the goodness of a design through moments of the number of coincidences between the rows of its projection designs. The new criterion is used to rank and classify designs of 16, 20, and 27 runs. Examples are given to illustrate that the ranking of designs is supported by other design criteria.
\end{abstract}

KEY WORDS: Generalized minimum aberration; Hadamard matrix; Hamming distance; Orthogonal array; Plackett-Burman design; Projection property.

\section{INTRODUCTION}

Consider a door panel stamping experiment for investigating the effects of the following seven factors on the formability of a panel: (A) concentration of lubricant, (B) panel thickness, (C) force on the outer portion of the door, (D) force on the inner portion of the door, (E) punch speed, $(\mathrm{F})$ thickness of lubrication, and $(\mathrm{G})$ manufacturers of lubricant. Each factor has two levels. The engineer plans to conduct a 20-run experiment due to budget limitation. He could choose seven columns from the 20-run Plackett-Burman design (Plackett and Burman 1946). Because there are many choices, he faces a practical question: How to select a design with good design properties?

The selection of fractional factorial designs for the problem described in the previous paragraph has always been an important issue in the area of experimental design. Two-level and three-level fractional factorial designs are frequently used in various scientific investigations. A regular design is determined by a defining relation (see, e.g., Box and Hunter 1961) and hence has a simple aliasing structure in that any two effects are either orthogonal or fully aliased. The run size is always a power of 2 or 3 , and thus the "gaps" between possible run sizes are getting wider as the power increases. Box and Hunter (1961) first introduced the concept of resolution for a regular fractional factorial design. Fries and Hunter (1980) proposed a refined criterion called minimum aberration to further differentiate designs with the same resolution. There are many additional works on minimum aberration designs (see $\mathrm{Wu}$ and Hamada 2000 for further references).

Nonregular factorial designs are commonly obtained from Plackett-Burman designs or Hadamard matrices in general by selecting a subset of the columns, which forms a projection. A Hadamard matrix of order $N$ is an $N \times N$ matrix with the elements \pm 1 whose columns (and rows) are orthogonal to each other. One can always "normalize" a Hadamard matrix by sign changes within complete rows so that its first column consists of all 1's. Removing the first column, one obtains a saturated two-level orthogonal array (OA) with $N$ runs and $N-1$ columns, which is a nonregular design if $N$ is a multiple of 4 but not a power of 2 . Other widely used nonregular designs are three-level and mixed-level OAs, as described by Dey and Mukerjee (1999), Hedayat, Sloane, and Stufken (1999), and Wu and Hamada (2000). Nonregular designs are useful for factor screening, and they fill the gaps between regular designs in terms of various run sizes. Unlike regular designs, nonregular designs exhibit a complex aliasing structure; that is, there exists at least one pair of effects that are neither orthogonal nor fully aliased. One can argue that this property is not a drawback. For regular designs, once the effects are confounded, they cannot be separated. For nonregular designs, if the effects are partially confounded, they may be simultaneously estimable. Hamada and $\mathrm{Wu}$ (1992) proposed an analysis strategy to turn the liability of complex aliasing structure into the virtue of model estimability (see Wu and Hamada 2000, chap. 8, for further references).

Nonregular factorial designs had not received sufficient attention due to their complex aliasing structure until recently. In the last decade, numerous authors studied the projection properties of two-level nonregular designs (see, e.g., Lin and Draper 1992; Wang and Wu 1995; Cheng 1995, 1998; Box and Tyssedal 1996; Dean and Draper 1999). More recently, Deng and Tang $(1999,2002)$ proposed generalized resolution and generalized minimum aberration for ranking nonregular twolevel designs in a systematic way. Their work shows a promising direction in the study of nonregular designs; however, their approach works only for two-level designs.

In this article, we propose a novel criterion, called moment aberration projection (MAP), to rank and classify nonregular designs (including multilevel designs). The key innovation is to investigate the relationship between the runs (i.e., rows), instead of the relationship between the factors (i.e., columns). For each design, we consider its projection designs and compute the power moments of the number of coincidences between the rows of its projection designs. The new criterion then assesses the goodness of designs by comparing the distribution of the power moments.

(C) 2005 American Statistical Association and the American Society for Quality TECHNOMETRICS, MAY 2005, VOL. 47, NO. 2 DOI 10.1198/004017004000000617 
The number of noncoincidences is called Hamming distance and has been studied extensively in the area of coding theory. Recently it has become popular in the area of design of experiment. Clark and Dean (2001) and Ma, Fang, and Lin (2001) studied the isomorphism of fractional factorial designs by means of the Hamming distances between the rows of their projection designs. Two designs are isomorphic (or equivalent) if one can be obtained from the other by permuting the rows, the columns, and the levels of each column. Clark and Dean (2001) proposed an algorithm for verifying the equivalence or nonequivalence of two-level designs and also developed some theoretical results on the equivalence of multilevel designs. Ma et al. (2001) proposed an algorithm for detecting nonequivalence of multilevel designs, and Ma and Fang (2001) proposed a criterion based on Hamming distances to rank multilevel designs. Although it is important to know whether two designs are nonequivalent, it is more important to rank them. Our primary goal here is ranking designs, and classification is secondary.

Section 2 describes the MAP criterion and its connections with other familiar criteria. Section 3 applies MAP to search for good designs for 16, 20, and 27 runs. For 16- and 20-run designs, the rankings by MAP and generalized minimum aberration are fairly consistent, but not identical. Nevertheless, the former has a better classification power than the latter. In particular, MAP can completely classify all 16-run OAs. For 27 runs, many nonregular designs are found to have better projection properties than regular designs. Section 4 studies the design selection for the door panel stamping experiment. Other criteria, such as estimation capacity and projection properties, are used to evaluate designs. Empirical studies show that the MAP ranking is fairly consistent with the number of estimable models and is supported by estimation capacity and hidden projection properties. Section 5 gives concluding remarks.

\section{MOMENT ABERRATION PROJECTION}

\subsection{Power Moments and Moment Aberration}

A design of $N$ runs and $m$ factors is represented by an $N \times m$ matrix, where each row corresponds to a run (i.e., treatment) and each column corresponds to a factor. A design has $s$ levels if each column takes on $s$ different values. For an $N \times m$ design d and a positive integer $t$, define the $t$ th power moment as

$$
K_{t}(\mathbf{d})=\sum_{1 \leq i<j \leq N}\left[\delta_{i j}(\mathbf{d})\right]^{t},
$$

where $\delta_{i j}(\mathbf{d})$ is the number of coincidences between the $i$ th and $j$ th rows. For two row vectors $\left(x_{1}, \ldots, x_{m}\right)$ and $\left(y_{1}, \ldots, y_{m}\right)$, the number of coincidences is the number of $i$ 's such that $x_{i}=y_{i}$.

The power moments measure the similarity among runs (i.e., rows). The first and second power moments measure the average and variance of the similarity among runs. Minimizing the power moments makes runs as dissimilar as possible. Therefore, good designs should have small power moments. This leads to the minimum moment aberration criterion (Xu 2003), which is used to sequentially minimize the power moments $K_{1}, K_{2}, \ldots$.

Although computation of the power moments involves the number of coincidences between rows, the power moments also measure the orthogonality among columns. This important observation was first made by Xu (2003). Specifically, for an $N \times m$ design $\mathbf{d}$ with $s$ levels, Xu showed that

$$
K_{1}(\mathbf{d}) \geq \frac{N m(N-s)}{2 s},
$$

with equality if and only if every column of $\mathbf{d}$ is balanced (i.e., each level appears equally often), and

$$
K_{2}(\mathbf{d}) \geq \frac{N m\left(N(m+s-1)-m s^{2}\right)}{2 s^{2}},
$$

with equality if and only if $\mathbf{d}$ is an OA. Note that $K_{t}$ defined in (1) is $N(N-1) / 2$ times that defined by Xu (2003). Based on a generalization of (3), Xu (2002) developed an efficient algorithm for constructing OAs and nearly-OAs with a small number of runs.

Example 1. Consider two $4 \times 3$ designs,

$$
\mathbf{d}_{1}=\left(\begin{array}{ccc}
+ & + & + \\
+ & - & - \\
- & + & - \\
- & - & +
\end{array}\right) \quad \text { and } \quad \mathbf{d}_{2}=\left(\begin{array}{ccc}
+ & + & + \\
- & + & + \\
- & - & + \\
- & - & -
\end{array}\right) \text {. }
$$

The first design, $\mathbf{d}_{1}$, is a $2^{3-1}$ regular fractional factorial design, and the second design, $\mathbf{d}_{2}$, is a one-factor-at-a-time design. Their coincidence matrices $\left(\delta_{i j}\right)$ are

$$
\left(\begin{array}{llll}
3 & 1 & 1 & 1 \\
1 & 3 & 1 & 1 \\
1 & 1 & 3 & 1 \\
1 & 1 & 1 & 3
\end{array}\right) \quad \text { and } \quad\left(\begin{array}{llll}
3 & 2 & 1 & 0 \\
2 & 3 & 2 & 1 \\
1 & 2 & 3 & 2 \\
0 & 1 & 2 & 3
\end{array}\right)
$$

The $t$ th power moment, $K_{t}$, is the sum of the $t$ th power of the elements above the diagonal of the coincidence matrix. It is easy to verify that $K_{t}\left(\mathbf{d}_{1}\right)=6$ for all $t \geq 1$ and $K_{1}\left(\mathbf{d}_{2}\right)=8, K_{2}\left(\mathbf{d}_{2}\right)=14, K_{3}\left(\mathbf{d}_{2}\right)=26$, and so on. It is evident that $K_{1}\left(\mathbf{d}_{1}\right)<K_{2}\left(\mathbf{d}_{2}\right)$ for all $t \geq 1$. Therefore, $\mathbf{d}_{1}$ has less moment aberration than $\mathbf{d}_{2}$ and is preferred. This agrees with the well-known fact that fractional factorial designs are superior to one-factor-at-a-time designs. Note that the lower bounds in (2) and (3) are achieved for $\mathbf{d}_{1}$, because it is an OA.

It is important to note the connection between designs in statistics and codes in coding theory. (For an introduction and applications to OAs, see Hedayat et al. 1999, chap. 4.) The Hamming distance between a pair of rows is the number of places where they differ. Let $h_{i j}(\mathbf{d})$ be the Hamming distance between the $i$ th and $j$ th rows. Evidently, $h_{i j}(\mathbf{d})=m-\delta_{i j}(\mathbf{d})$. Let $B_{k}(\mathbf{d})$ be the number of pairs of rows of $\mathbf{d}$ such that their Hamming distance is equal to $k$, that is, $B_{k}(\mathbf{d})=\mid\{(i, j)$ : $\left.h_{i j}(\mathbf{d})=k, 1 \leq i<j \leq N\right\} \mid$. It is evident that

$$
K_{t}(\mathbf{d})=\sum_{i=0}^{m}(m-i)^{t} B_{i}(\mathbf{d}) .
$$

This equation implies that the first $m$ power moments uniquely determine the rest and thus at most $m$ comparisons are necessary in the moment aberration criterion.

By applying MacWilliams identities and Pless power moment identities (see, e.g., MacWilliams and Sloane 1977, chap. 5), two fundamental results in coding theory, Xu (2003) showed that minimum moment aberration is equivalent to minimum aberration (Fries and Hunter 1980) for regular designs 
and to generalized minimum aberration (GMA) (Xu and $\mathrm{Wu}$ 2001; Ma and Fang 2001) for nonregular designs.

The moment aberration criterion can also be applied to supersaturated designs. A design is supersaturated if it does not have sufficient degrees of freedom to estimate all main effects simultaneously. A number of criteria have been proposed. Xu (2003) showed that the minimization of $K_{2}$ is equivalent to the minimization of $E\left(s^{2}\right)$ (Booth and Cox 1962) for two-level designs and the minimization of average chi-squared (Yamada and Lin 1999) for three-level designs.

\subsection{Moment Aberration Projection}

Consider the projection designs of an $N \times m$ design d. For each projection design, we can compute the power moments as in (1) for any $t$. Instead of using all the power moments, we propose simply using one moment for each projection, because it is cheaper to compute and compare a number than a vector. Specifically, we use the $p$ th moment $K_{p}$ for a $p$-factor projection, that is, let $t=p$ in (1). For given $p(1 \leq p \leq m)$, there are $\left(\begin{array}{l}m \\ p\end{array}\right) p$-factor projections. The frequency distribution of $K_{p}$-values of these projections is called the p-dimensional $K$-value distribution and is denoted by $\mathbf{F}_{p}(\mathbf{d})$.

Let $\mathbf{F}=\left(f\left(x_{1}\right), \ldots, f\left(x_{c}\right)\right)$ and $\mathbf{G}=\left(g\left(x_{1}\right), \ldots, g\left(x_{c}\right)\right)$ be two frequency distributions taking $c$ possible values (in decreasing order) on $x_{1}>\cdots>x_{c}$. We would sequentially compare the frequency of the components and prefer the one that minimizes the frequency of the largest value. For convenience, we write $\mathbf{F}<\mathbf{G}$ if there exists an index $i(1 \leq i \leq c)$ such that $f\left(x_{i}\right)<g\left(x_{i}\right)$ and $f\left(x_{j}\right)=g\left(x_{j}\right)$ for all $j<i$.

For two $N \times m$ designs $\mathbf{d}_{1}$ and $\mathbf{d}_{2}$, suppose that $p$ is the smallest integer such that the $p$-dimensional $K$-value distributions are different, that is, $\mathbf{F}_{p}\left(\mathbf{d}_{1}\right) \neq \mathbf{F}_{p}\left(\mathbf{d}_{2}\right)$. We say that $\mathbf{d}_{1}$ has less MAP than $\mathbf{d}_{2}$ if $\mathbf{F}_{p}\left(\mathbf{d}_{1}\right)<\mathbf{F}_{p}\left(\mathbf{d}_{2}\right)$.

Example 2 (Continued from Example 1). For $\mathbf{d}_{1}$, each column forms a one-factor projection with $K_{1}=2$, each pair of columns forms a two-factor projection with $K_{2}=4$, and all three columns together form a three-factor projection (i.e., $\mathbf{d}_{1}$ itself) with $K_{3}=6$. For $\mathbf{d}_{2}$, the second column forms a one-factor projection with $K_{1}=2$ and the other two one-factor projections with $K_{1}=3$, the first and third columns form a two-factor projection with $K_{2}=6$ and the other two two-factor projections with $K_{2}=5$, and all three columns together form a three-factor projection (i.e., $\mathbf{d}_{2}$ itself) with $K_{3}=26$. In summary, the $K$-value distributions are as follows:

\begin{tabular}{lccc}
\hline \hline Design & $\mathbf{F}_{1}:(3,2)$ & $\mathbf{F}_{2}:(6,5,4)$ & $\mathbf{F}_{3}:(26,6)$ \\
\hline $\mathbf{d}_{1}$ & $(0,3)$ & $(0,0,3)$ & $(0,1)$ \\
$\mathbf{d}_{2}$ & $(2,1)$ & $(1,2,0)$ & $(1,0)$ \\
\hline
\end{tabular}

It is evident that $\mathbf{F}_{p}\left(\mathbf{d}_{1}\right)<\mathbf{F}_{p}\left(\mathbf{d}_{2}\right)$ for $p=1,2,3$. Therefore, according to MAP, $\mathbf{d}_{1}$ is better than $\mathbf{d}_{2}$.

Example 3. Consider the 12-run Plackett-Burman design given in Table 1 . This design is constructed by cyclically shifting the first row $(+,+,-,+,+,+,-,-,-,+,-)$ to the right 10 times and adding a row of -'s (Plackett and Burman 1946). According to Lin and Draper (1992), there are
Table 1. The 12-Run Plackett-Burman Design

\begin{tabular}{rrrrrrrrrrrr}
\hline \hline Run & 1 & 2 & 3 & 4 & 5 & 6 & 7 & 8 & 9 & 10 & 11 \\
\hline 1 & + & + & - & + & + & + & - & - & - & + & - \\
2 & - & + & + & - & + & + & + & - & - & - & + \\
3 & + & - & + & + & - & + & + & + & - & - & - \\
4 & - & + & - & + & + & - & + & + & + & - & - \\
5 & - & - & + & - & + & + & - & + & + & + & - \\
6 & - & - & - & + & - & + & + & - & + & + & + \\
7 & + & - & - & - & + & - & + & + & - & + & + \\
8 & + & + & - & - & - & + & - & + & + & - & + \\
9 & + & + & + & - & - & - & + & - & + & + & - \\
10 & - & + & + & + & - & - & - & + & - & + & + \\
11 & + & - & + & + & + & - & - & - & + & - & + \\
12 & - & - & - & - & - & - & - & - & - & - & - \\
\hline
\end{tabular}

two nonisomorphic $12 \times 5$ projection designs: design 5.1 and design 5.2. For example, columns $1-4$ and 10 form design 5.1 and columns 1-5 form design 5.2. The former has two repeated runs, whereas the latter has two mirror image runs. From (2) and (3), any one-factor projection must have $K_{1}=30$ and any two-factor projection must have $K_{2}=84$, because both designs are OAs. The three-, four-, and five-dimensional $K$-value distributions are as follows:

\begin{tabular}{lccc}
\hline \hline Design & $\mathbf{F}_{3}: 330$ & $\mathbf{F}_{4}: 1,728$ & $\mathbf{F}_{5}:(11,070,10,950)$ \\
\hline 5.1 & 10 & 5 & $(0,1)$ \\
5.2 & 10 & 5 & $(1,0)$ \\
\hline
\end{tabular}

We see that for both designs, all 10 three-factor projections have $K_{3}=330$, and all five four-factor projections have $K_{4}=1,728$. This is because all three-factor projections are equivalent and so are all four-factor projections (Lin and Draper 1992). Therefore, they are not distinguishable when projected onto three and four factors. Nevertheless, they are distinguishable when projected onto five factors because $K_{5}=10,950$ for design 5.1 and $K_{5}=11,070$ for design 5.2. Therefore, according to MAP, design 5.1 is better than design 5.2, which is consistent with the conclusions of Wang and Wu (1995) and Deng and Tang (1999).

The MAP is closely related to the GMA criterion proposed by Deng and Tang $(1999,2002)$. For an $N \times m$ design $\mathbf{d}=\left(x_{i j}\right)$ with entries -1 or +1 , define

$$
J_{m}(\mathbf{d})=\left|\sum_{i=1}^{N} x_{i 1} x_{i 2} \cdots x_{i m}\right| .
$$

The quantity $J_{m}(\mathbf{d})$ measures the correlations among the columns for two-level designs. The $p$-dimensional $J$-value distribution, called the confounding frequency vector by Deng and Tang, is the frequency distribution of $J_{p}$-values of the $p$-factor projections. The GMA criterion sequentially minimizes the confounding frequency vectors as MAP does. Because both GMA and MAP first consider lower-dimensional projections and then proceed to higher-dimensional projections, we would expect them to produce consistent results, as was demonstrated in our study for designs of 12,16 , and 20 runs.

One important advantage of MAP over GMA is that the former works for both two-level and multilevel designs, whereas the latter works only for two-level designs. Here is an example for three-level designs. 
Example 4. Consider the commonly used $O A\left(18,3^{7}\right)$ given in Table 2. Wang and Wu (1995) showed that there are four nonisomorphic four-factor projections. Let design 4.1 consist of columns 2-5, let design 4.2 consist of columns $1-3$ and 6 , let design 4.3 consist of columns $1-4$, and let design 4.4 consist of columns 1, 2, 5, and 7. From (2) and (3), any one-factor projection must have $K_{1}=45$, and any two-factor projection must have $K_{2}=108$, because all designs are OAs. The threeand four-dimensional $K$-value distributions are as follows:

\begin{tabular}{lcc}
\hline \hline Design & $\mathbf{F}_{3}:(351,315,297)$ & $\mathbf{F}_{4}:(1,260,1,044,936)$ \\
\hline 4.1 & $(0,0,4)$ & $(0,0,1)$ \\
4.2 & $(0,1,3)$ & $(0,1,0)$ \\
4.3 & $(1,0,3)$ & $(1,0,0)$ \\
4.4 & $(1,3,0)$ & $(1,0,0)$ \\
\hline
\end{tabular}

When projected onto three factors, all four projections of design 4.1 have $K_{3}=297$, three projections of design 4.2 have $K_{3}=297$ and one projection has $K_{3}=315$, three projections of design 4.3 have $K_{3}=297$ and one projection has $K_{3}=351$, and three projections of design 4.4 have $K_{3}=315$ and one projection has $K_{3}=351$. Therefore, according to MAP, design 4.1 is better than design 4.2 , which in turn is better than designs 4.3 and 4.4. This ranking is consistent with that of Wang and $\mathrm{Wu}$ (1995). It is important to note that the moment aberration criterion is not able to distinguish between design 4.3 and design 4.4. In contrast, according to MAP, design 4.3 is better than design 4.4, a result supported by Wang and Wu (1995) in terms of their hidden projection properties.

As the previous example demonstrates, the frequency distribution $\mathbf{F}_{p}(\mathbf{d})$ provides a quick method for checking whether two designs are nonisomorphic. A necessary condition for isomorphism of two designs is that they have the same $p$-dimensional $K$-value distribution, $\mathbf{F}_{p}(\mathbf{d})$, for all dimension $p(1 \leq p \leq m)$. Whenever two designs have different $K$-value distributions, we can declare that they are not isomorphic. The same argument works for GMA based on the $J$-values defined in (5). However, MAP has a much better classification power than GMA (see the next section for examples). The reason is that $K_{t}$ defined in (1) takes on many more values than $J_{m}$ defined in (5). Deng

Table 2. An 18-Run Orthogonal Array

\begin{tabular}{rlllllll}
\hline \hline Run & 1 & 2 & 3 & 4 & 5 & 6 & 7 \\
\hline 1 & 0 & 0 & 0 & 0 & 0 & 0 & 0 \\
2 & 0 & 1 & 1 & 1 & 1 & 1 & 1 \\
3 & 0 & 2 & 2 & 2 & 2 & 2 & 2 \\
4 & 1 & 0 & 0 & 1 & 1 & 2 & 2 \\
5 & 1 & 1 & 1 & 2 & 2 & 0 & 0 \\
6 & 1 & 2 & 2 & 0 & 0 & 1 & 1 \\
7 & 2 & 0 & 1 & 0 & 2 & 1 & 2 \\
8 & 2 & 1 & 2 & 1 & 0 & 2 & 0 \\
9 & 2 & 2 & 0 & 2 & 1 & 0 & 1 \\
10 & 0 & 0 & 2 & 2 & 1 & 1 & 0 \\
11 & 0 & 1 & 0 & 0 & 2 & 2 & 1 \\
12 & 0 & 2 & 1 & 1 & 0 & 0 & 2 \\
13 & 1 & 0 & 1 & 2 & 0 & 2 & 1 \\
14 & 1 & 1 & 2 & 0 & 1 & 0 & 2 \\
15 & 1 & 2 & 0 & 1 & 2 & 1 & 0 \\
16 & 2 & 0 & 2 & 1 & 2 & 0 & 1 \\
17 & 2 & 1 & 0 & 2 & 0 & 1 & 2 \\
18 & 2 & 2 & 1 & 0 & 1 & 2 & 0 \\
\hline
\end{tabular}

and Tang (2002) showed that $J_{m}(\mathbf{d})$ takes on only a few values between 0 and $N$.

Clark and Dean (2001) proposed two algorithms, Deseq 1 and Deseq2, for checking the equivalence of two-level fractional factorial designs. Deseq 1 does an initial check for nonequivalence, and Deseq 2 looks for row and column permutations if two designs are isomorphic. It is of interest to compare MAP with Deseq 1 because both provide sufficient (but not necessary) conditions for nonisomorphism and can be used as a preliminary step to identify nonisomorphic designs. One difference is that for each projection design, Deseq 1 uses the frequency distribution of Hamming distances between all possible pairs of rows, whereas MAP combines the numbers in the calculation of $K$ and uses only one $K$ value. It is certainly neater to compare and rank two designs using two numbers than to use two frequency distributions of Hamming distances. Our empirical study suggested that using one $K$ value usually does not lose much, if any, information.

When restricted to two-level or multilevel regular designs, GMA and MAP are equivalent to minimum aberration. It is interesting to note that MAP is not equivalent to minimum aberration. For example, consider two regular $2^{12-3}$ designs given by Draper and Mitchell (1968), labeled designs 3.4 and 3.5 in their table 1. These two designs are not isomorphic but share the same word-length pattern; therefore, they are equivalent under the minimum aberration criterion. On the other hand, it is straightforward to verify that their 9-, 10-, and 11-dimensional $K$-value distributions are different; therefore, they are not equivalent under MAP.

\section{RANKING AND CLASSIFICATION WITH MOMENT ABERRATION PROJECTION}

\subsection{Designs of 16 Runs}

According to Hall (1961), there are precisely five nonisomorphic Hadamard matrices, labeled I, II, III, IV, and V. In particular, type $\mathrm{I}$ is a regular design and is equivalent to the 16-run Plackett-Burman design, whose cyclic generator is $(+,+,+,+,-,+,-,+,+,-,-,+,-,-,-)$. For convenience, we use the Plackett-Burman design for type I. Deng and Tang (2002) used GMA to rank and classify designs from the five Hadamard designs; here we use MAP to reexamine these designs.

For each $m, 3 \leq m \leq 15$, we use MAP to rank and classify all nonequivalent designs from the five Hadamard matrices by searching over all $m$-factor projections. Table 3 lists a few top designs ranked by MAP, their three- and four-dimensional $K$-value distributions, GMA rankings from Deng and Tang (2002), types, and the corresponding columns. A design of $m$ factors is denoted by $16 . m . i$, where $i$ denotes the rank under MAP. As we can see from Table 3, the rankings by MAP and GMA are quite consistent. Both criteria identify the same top designs, except for two cases where MAP further distinguishes between the top designs ranked by GMA (designs 16.12.1 and 16.12.2 and designs 16.15.1 and 16.15.2).

As a byproduct, we investigate the equivalence of projection designs from the five Hadamard matrices. Table 4 shows the number of nonisomorphic projection designs identified by 
Table 3. Some 16-Run Designs Ranked by MAP

\begin{tabular}{|c|c|c|c|c|c|}
\hline Design & $\boldsymbol{F}_{3}$ & $F_{4}$ & $G M A$ & Type & Columns \\
\hline $\begin{array}{l}16.3 .1 \\
16.3 .2 \\
16.3 .3\end{array}$ & $\begin{array}{l}(0,0,1) \\
(0,1,0) \\
(1,0,0)\end{array}$ & & $\begin{array}{l}1 \\
2 \\
3\end{array}$ & $\begin{array}{c}1 \\
11 \\
11\end{array}$ & $\begin{array}{c}123 \\
4812 \\
1213\end{array}$ \\
\hline $\begin{array}{l}16.4 .1 \\
16.4 .2 \\
16.4 .3\end{array}$ & $\begin{array}{l}(0,0,4) \\
(0,0,4) \\
(0,1,3)\end{array}$ & $\begin{array}{l}(0,0,0,0,1) \\
(0,0,0,1,0) \\
(0,0,1,0,0)\end{array}$ & $\begin{array}{l}1 \\
2 \\
3\end{array}$ & $\begin{array}{l}1 \\
1\end{array}$ & $\begin{array}{c}1234 \\
1238 \\
14812\end{array}$ \\
\hline $\begin{array}{l}16.5 .1 \\
16.5 .2 \\
16.5 .3\end{array}$ & $\begin{array}{c}(0,0,10) \\
(0,0,10) \\
(0,1,9)\end{array}$ & $\begin{array}{l}(0,0,0,0,5) \\
(0,0,0,1,4) \\
(0,0,2,0,3)\end{array}$ & $\begin{array}{l}1 \\
2 \\
3\end{array}$ & $\begin{array}{l}1 \\
1\end{array}$ & $\begin{array}{c}12347 \\
12346 \\
124812\end{array}$ \\
\hline $\begin{array}{l}16.6 .1 \\
16.6 .2 \\
16.6 .3 \\
16.6 .4\end{array}$ & $\begin{array}{l}(0,0,20) \\
(0,2,18) \\
(0,4,16) \\
(0,4,16)\end{array}$ & $\begin{array}{c}(0,0,0,3,12) \\
(0,1,4,1,9) \\
(0,3,6,0,6) \\
(0,4,4,0,7)\end{array}$ & $\begin{array}{l}1 \\
2 \\
4 \\
3\end{array}$ & $\begin{array}{l}\text { I } \\
\text { II } \\
\text { III } \\
\text { II }\end{array}$ & $\begin{array}{c}123468 \\
1247812 \\
12481012 \\
14681112\end{array}$ \\
\hline $\begin{array}{l}16.7 .1 \\
16.7 .2 \\
16.7 .3\end{array}$ & $\begin{array}{l}(0,0,35) \\
(0,4,31) \\
(0,6,29)\end{array}$ & $\begin{array}{c}(0,0,0,7,28) \\
(0,4,8,3,20) \\
(0,6,12,1,16)\end{array}$ & $\begin{array}{l}1 \\
2 \\
3\end{array}$ & $\begin{array}{l}\text { I } \\
\text { II } \\
\text { III }\end{array}$ & $\begin{array}{c}1234689 \\
124781112 \\
124781012\end{array}$ \\
\hline $\begin{array}{l}16.8 .1 \\
16.8 .2 \\
16.8 .3 \\
16.8 .4\end{array}$ & $\begin{array}{l}(0,0,56) \\
(0,8,48) \\
(0,12,44) \\
(0,12,44)\end{array}$ & $\begin{array}{c}(0,0,0,14,56) \\
(0,12,16,6,36) \\
(0,18,24,1,27) \\
(0,18,24,1,27)\end{array}$ & $\begin{array}{l}1 \\
2 \\
3 \\
3\end{array}$ & $\begin{array}{l}\text { I } \\
\text { II } \\
\text { V } \\
\text { III }\end{array}$ & $\begin{aligned} 123468912 \\
12478111215 \\
12478101215 \\
12478101215\end{aligned}$ \\
\hline $\begin{array}{l}16.9 .1 \\
16.9 .2 \\
16.9 .3\end{array}$ & $\begin{array}{l}(0,16,68) \\
(0,20,64) \\
(0,22,62)\end{array}$ & $\begin{array}{l}(0,48,0,14,64) \\
(0,48,24,6,48) \\
(0,48,36,2,40)\end{array}$ & $\begin{array}{l}1 \\
2 \\
3\end{array}$ & $\begin{array}{l}\text { II } \\
\text { III } \\
\text { V }\end{array}$ & $\begin{array}{l}456789101112 \\
234589101112 \\
124789101214\end{array}$ \\
\hline $\begin{array}{l}16.10 .1 \\
16.10 .2 \\
16.10 .3\end{array}$ & $\begin{array}{l}(0,32,88) \\
(0,32,88) \\
(0,32,88)\end{array}$ & $\begin{array}{c}(0,96,32,10,72) \\
(0,104,16,14,76) \\
(0,112,0,18,80)\end{array}$ & $\begin{array}{l}1 \\
2 \\
3\end{array}$ & $\begin{array}{l}\text { III } \\
\text { III } \\
\text { II }\end{array}$ & $\begin{array}{c}23458910111213 \\
2489101112131415 \\
45678910111213\end{array}$ \\
\hline $\begin{array}{l}16.11 .1 \\
16.11 .2 \\
16.11 .3 \\
16.11 .4 \\
16.11 .5\end{array}$ & $\begin{array}{l}(0,48,117) \\
(0,48,117) \\
(0,48,117) \\
(0,48,117) \\
(0,48,117)\end{array}$ & $\begin{array}{c}(0,156,72,8,94) \\
(0,160,64,10,96) \\
(0,168,48,14,100) \\
(0,168,48,14,100) \\
(0,168,48,14,100)\end{array}$ & $\begin{array}{l}1 \\
2 \\
3 \\
3 \\
3\end{array}$ & $\begin{array}{l}\text { V } \\
\text { IV } \\
\text { III } \\
\text { V } \\
\text { III }\end{array}$ & $\begin{array}{c}1247891011121314 \\
23456789101112 \\
24789101112131415 \\
12489101112131415 \\
2345891011121314\end{array}$ \\
\hline $\begin{array}{l}16.12 .1 \\
16.12 .2 \\
16.12 .3\end{array}$ & $\begin{array}{l}(0,64,156) \\
(0,64,156) \\
(0,64,156)\end{array}$ & $\begin{array}{l}(0,240,96,15,144) \\
(0,240,96,15,144) \\
(0,256,64,23,152)\end{array}$ & $\begin{array}{l}1 \\
1 \\
2\end{array}$ & $\begin{array}{l}\text { V } \\
\text { IV } \\
\text { III }\end{array}$ & $\begin{array}{c}124789101112131415 \\
2345678910111213 \\
234589101112131415\end{array}$ \\
\hline $\begin{array}{l}16.13 .1 \\
16.13 .2 \\
16.13 .3\end{array}$ & $\begin{array}{l}(0,88,198) \\
(2,80,204) \\
(2,80,204)\end{array}$ & $\begin{array}{l}(0,360,160,15,180) \\
(20,320,160,15,200) \\
(20,336,128,23,208)\end{array}$ & $\begin{array}{l}1 \\
2 \\
3\end{array}$ & $\begin{array}{l}\text { IV } \\
\text { V } \\
\text { III }\end{array}$ & $\begin{array}{l}234567891011121314 \\
1234589101112131415 \\
2345689101112131415\end{array}$ \\
\hline $\begin{array}{l}16.14 .1 \\
16.14 .2 \\
16.14 .3\end{array}$ & $\begin{array}{l}(0,112,252) \\
(4,96,264) \\
(4,96,264)\end{array}$ & $\begin{array}{l}(0,504,224,21,252) \\
(44,408,240,17,292) \\
(44,432,192,29,304)\end{array}$ & $\begin{array}{l}1 \\
2 \\
3\end{array}$ & $\begin{array}{l}\text { IV } \\
\text { V } \\
\text { III }\end{array}$ & $\begin{array}{l}23456789101112131415 \\
12345689101112131415 \\
23456789101112131415\end{array}$ \\
\hline $\begin{array}{l}16.15 .1 \\
16.15 .2 \\
16.15 .3 \\
16.15 .4 \\
16.15 .5\end{array}$ & $\begin{array}{c}(7,112,336) \\
(7,112,336) \\
(11,96,348) \\
(19,64,372) \\
(35,0,420)\end{array}$ & $\begin{array}{c}(84,504,336,21,420) \\
(84,504,336,21,420) \\
(132,432,288,33,480) \\
(228,288,192,57,600) \\
(420,0,0,105,840)\end{array}$ & $\begin{array}{l}1 \\
1 \\
2 \\
3 \\
4\end{array}$ & $\begin{array}{l}\text { IV } \\
\text { V } \\
\text { III } \\
\text { II } \\
\text { I }\end{array}$ & 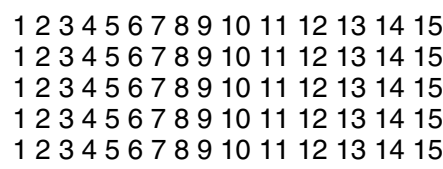 \\
\hline
\end{tabular}

NOTE: Type I is a Plackett-Burman design, and types II-V are as described by Deng and Tang (2002).

The indices of $\mathbf{F}_{3}$ (possible $K$ values) are $(744,672,648)$, and the indices of $\mathbf{F}_{4}$ are $(4,160,3,776,3,632,3,584,3,392)$.

MAP, GMA (Deng and Tang 2002), and a complete search of all possible permutations of rows, columns, and levels of each column (Sun 1993). The number of nonisomorphic designs found by MAP matches exactly with the complete search, whereas GMA found fewer designs for 6-12 and 15 columns. The conclusion is that MAP gives a complete classification for 16-run designs without a complete search of all possible permutations.

Table 4. The Number of 16-Run Nonisomorphic Designs

\begin{tabular}{lccccccccccccc}
\hline \hline Method & 3 & 4 & 5 & 6 & 7 & 8 & 9 & 10 & 11 & 12 & 13 & 14 & 15 \\
\hline MAP & 3 & 5 & 11 & 27 & 55 & 80 & 87 & 78 & 58 & 36 & 18 & 10 & 5 \\
GMA & 3 & 5 & 11 & 26 & 53 & 74 & 78 & 75 & 56 & 32 & 18 & 10 & 4 \\
Complete search & 3 & 5 & 11 & 27 & 55 & 80 & 87 & 78 & 58 & 36 & 18 & 10 & 5 \\
\hline
\end{tabular}

In fact, only projection dimension up to 7 is needed to classify all 16-run designs.

\subsection{Designs of 20 Runs}

Hall (1965) showed that there are three nonisomorphic Hadamard matrices, labeled Q, P, and N. Listings of these designs were given by Hall (1965) and by Deng and Tang (2002). As noted by Wang and $\mathrm{Wu}$ (1995), type $\mathrm{Q}$ is equivalent to the 20-run Plackett-Burman design, whose cyclic generator is $(+,+,-,-,+,+,+,+,-,+,-,+,-,-,-,-,+$, $+,-)$. For convenience, we use the Plackett-Burman design for type Q.

As before, for each $m, 3 \leq m \leq 19$, we use MAP to rank and classify all projection designs from the three Hadamard matrices by searching over all $m$-factor projections. It is possible 
that two nonisomorphic designs have the same $K$-value distributions. Table 5 lists a few top and bottom designs ranked by MAP; their three-, four-, and five-dimensional $K$-value distributions; GMA rankings from Deng and Tang (2002); types; and the corresponding columns. As we can see from Table 5, the rankings by MAP and GMA are still consistent, but the degree of consistency is less than for the 16-run case. For each $m$, the best design ranked by MAP, 20.m.1, is also ranked as the best by GMA. However, there are several cases where GMA fails to separate one or more of its top designs with 6-8 and 12-19 columns. In particular, MAP ranks three top GMA designs as 20.7.1, 20.7.2, and 20.7.8. In the next section we show that the MAP rankings are supported by other criteria.

Observe that the bottom design in Table 5 is from type $\mathrm{P}$ for 5-19 columns. Because our search goes through Q first, $\mathrm{P}$ next, and $\mathrm{N}$ last, this means that type $\mathrm{Q}$ does not contain the

Table 5. Some 20-Run Designs Ranked by MAP

\begin{tabular}{|c|c|c|c|c|c|c|}
\hline Design & $\boldsymbol{F}_{3}$ & $\boldsymbol{F}_{4}$ & $\boldsymbol{F}_{5}$ & $G M A$ & Type & Columns \\
\hline $\begin{array}{l}20.3 .1 \\
20.3 .2\end{array}$ & $\begin{array}{l}(0,1) \\
(1,0)\end{array}$ & & & $\begin{array}{l}1 \\
2\end{array}$ & $\begin{array}{l}Q \\
Q\end{array}$ & $\begin{array}{l}123 \\
129\end{array}$ \\
\hline $\begin{array}{l}20.4 .1 \\
20.4 .2 \\
20.4 .3\end{array}$ & $\begin{array}{l}(0,4) \\
(0,4) \\
(1,3)\end{array}$ & $\begin{array}{l}(0,0,1) \\
(0,1,0) \\
(1,0,0)\end{array}$ & & $\begin{array}{l}1 \\
2 \\
3\end{array}$ & $\begin{array}{l}Q \\
Q \\
Q\end{array}$ & $\begin{array}{c}1234 \\
12316 \\
1236\end{array}$ \\
\hline $\begin{array}{l}20.5 .1 \\
20.5 .2 \\
20.5 .3 \\
20.5 .10\end{array}$ & $\begin{array}{c}(0,10) \\
(0,10) \\
(0,10) \\
(2,8)\end{array}$ & $\begin{array}{l}(0,0,5) \\
(0,0,5) \\
(0,1,4) \\
(4,1,0)\end{array}$ & $\begin{array}{l}(0,0,0,0,0,0,0,0,0,1) \\
(0,0,0,0,0,0,0,0,1,0) \\
(0,0,0,0,0,0,0,1,0,0) \\
(1,0,0,0,0,0,0,0,0,0)\end{array}$ & $\begin{array}{r}1 \\
2 \\
3 \\
10\end{array}$ & $\begin{array}{l}Q \\
Q \\
Q \\
P\end{array}$ & $\begin{array}{c}12345 \\
123414 \\
123416 \\
12345\end{array}$ \\
\hline $\begin{array}{l}20.6 .1 \\
20.6 .2 \\
20.6 .3 \\
20.6 .4 \\
20.6 .59\end{array}$ & $\begin{array}{l}(0,20) \\
(0,20) \\
(0,20) \\
(0,20) \\
(4,16)\end{array}$ & $\begin{array}{l}(0,1,14) \\
(0,1,14) \\
(0,1,14) \\
(0,1,14) \\
(12,3,0)\end{array}$ & $\begin{array}{l}(0,0,0,0,0,0,0,2,2,2) \\
(0,0,0,0,0,0,0,2,3,1) \\
(0,0,0,0,0,0,1,1,2,2) \\
(0,0,0,0,0,0,2,0,0,4) \\
(3,3,0,0,0,0,0,0,0,0)\end{array}$ & $\begin{array}{r}1 \\
2 \\
2 \\
1 \\
34\end{array}$ & $\begin{array}{l}Q \\
Q \\
Q \\
Q \\
P\end{array}$ & $\begin{array}{rl}1 & 2341316 \\
1 & 234816 \\
1 & 234515 \\
1 & 234513 \\
1 & 2351015\end{array}$ \\
\hline $\begin{array}{l}20.7 .1 \\
20.7 .2 \\
20.7 .3 \\
20.7 .4 \\
20.7 .5 \\
20.7 .6 \\
20.7 .7 \\
20.7 .8 \\
20.7 .9 \\
20.7 .10 \\
20.7 .384 \\
20.7 .385 \\
20.7 .386 \\
20.7 .387 \\
20.7 .388\end{array}$ & $\begin{array}{l}(0,35) \\
(0,35) \\
(0,35) \\
(0,35) \\
(0,35) \\
(0,35) \\
(0,35) \\
(0,35) \\
(0,35) \\
(0,35) \\
(4,31) \\
(4,31) \\
(5,30) \\
(5,30) \\
(7,28)\end{array}$ & $\begin{array}{c}(0,3,32) \\
(0,3,32) \\
(0,3,32) \\
(0,3,32) \\
(0,3,32) \\
(0,3,32) \\
(0,3,32) \\
(0,3,32) \\
(0,4,31) \\
(0,4,31) \\
(16,4,15) \\
(16,4,15) \\
(20,3,12) \\
(20,5,10) \\
(28,7,0)\end{array}$ & $\begin{array}{c}(0,0,0,0,0,0,0,9,7,5) \\
(0,0,0,0,0,0,1,8,6,6) \\
(0,0,0,0,0,0,1,8,8,4) \\
(0,0,0,0,0,0,1,8,8,4) \\
(0,0,0,0,0,0,2,7,7,5) \\
(0,0,0,0,0,0,2,7,7,5) \\
(0,0,0,0,0,0,3,6,6,6) \\
(0,0,0,0,0,0,4,5,3,9) \\
(0,0,0,0,0,0,2,10,3,6) \\
(0,0,0,0,0,0,2,10,5,4) \\
(1,1,4,7,3,2,1,2,0,0) \\
(1,1,4,7,3,2,3,0,0,0) \\
(1,2,6,6,4,2,0,0,0,0) \\
(3,0,6,12,0,0,0,0,0,0) \\
(9,12,0,0,0,0,0,0,0,0)\end{array}$ & $\begin{array}{r}1 \\
1 \\
2 \\
2 \\
2 \\
2 \\
2 \\
1 \\
3 \\
4 \\
47 \\
48 \\
49 \\
50 \\
51\end{array}$ & $\begin{array}{l}P \\
Q \\
P \\
Q \\
Q \\
P \\
Q \\
Q \\
Q \\
P \\
P \\
P \\
P \\
P \\
P\end{array}$ & 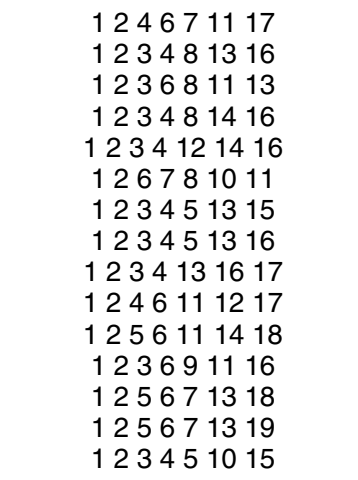 \\
\hline $\begin{array}{l}20.8 .1 \\
20.8 .2 \\
20.8 .3 \\
20.8 .1265\end{array}$ & $\begin{array}{l}(0,56) \\
(0,56) \\
(0,56) \\
(8,48)\end{array}$ & $\begin{array}{l}(0,6,64) \\
(0,6,64) \\
(0,8,62) \\
(40,10,20)\end{array}$ & $\begin{array}{c}(0,0,0,0,0,0,4,20,20,12) \\
(0,0,0,0,0,0,4,20,20,12) \\
(0,0,0,0,0,0,6,26,10,14) \\
(8,0,16,32,0,0,0,0,0,0)\end{array}$ & $\begin{array}{r}1 \\
1 \\
2 \\
80\end{array}$ & $\begin{array}{l}N \\
P \\
P \\
P\end{array}$ & $\begin{array}{c}1234681618 \\
126810111315 \\
1234671117 \\
5671011121516\end{array}$ \\
\hline $\begin{array}{l}20.9 .1 \\
20.9 .2 \\
20.9 .3 \\
20.9 .2089\end{array}$ & $\begin{array}{l}(0,84) \\
(0,84) \\
(1,83) \\
(12,72)\end{array}$ & $\begin{array}{l}(0,18,108) \\
(0,18,108) \\
(6,14,106) \\
(72,18,36)\end{array}$ & $\begin{array}{c}(0,0,0,0,0,0,18,72,16,20) \\
(0,0,0,0,0,0,18,72,36,0) \\
(0,0,0,4,2,9,13,53,19,26) \\
(18,0,36,72,0,0,0,0,0,0)\end{array}$ & $\begin{array}{r}1 \\
2 \\
3 \\
125\end{array}$ & $\begin{array}{l}\mathrm{P} \\
\mathrm{P} \\
\mathrm{P} \\
\mathrm{P}\end{array}$ & $\begin{array}{c}123489131418 \\
1238913141819 \\
123467111216 \\
567101112151617\end{array}$ \\
\hline $\begin{array}{l}20.10 .1 \\
20.10 .2 \\
20.10 .3 \\
20.10 .2282\end{array}$ & $\begin{array}{l}(0,120) \\
(2,118) \\
(3,117) \\
(12,108)\end{array}$ & $\begin{array}{l}(0,30,180) \\
(14,22,174) \\
(21,18,171) \\
(84,18,108)\end{array}$ & $\begin{array}{c}(0,0,0,0,0,0,36,144,36,36) \\
(0,0,0,12,6,24,24,96,42,48) \\
(0,0,0,18,9,36,18,72,45,54) \\
(18,0,36,72,16,56,2,16,20,16)\end{array}$ & $\begin{array}{r}1 \\
2 \\
3 \\
125\end{array}$ & $\begin{array}{l}P \\
P \\
Q \\
P\end{array}$ & $\begin{array}{r}12348913141819 \\
12346711121617 \\
12346813141617 \\
1567101112151617\end{array}$ \\
\hline $\begin{array}{l}20.11 .1 \\
20.11 .2 \\
20.11 .3 \\
20.11 .1899\end{array}$ & $\begin{array}{l}(5,160) \\
(6,159) \\
(6,159) \\
(13,152)\end{array}$ & $\begin{array}{c}(40,30,260) \\
(48,26,256) \\
(48,26,256) \\
(104,26,200)\end{array}$ & $\begin{array}{c}(6,0,4,8,16,96,36,160,84,52) \\
(0,7,0,28,28,98,28,119,84,70) \\
(2,4,4,22,29,97,33,121,76,74) \\
(22,32,0,0,48,208,24,104,24,0)\end{array}$ & $\begin{array}{r}1 \\
2 \\
2 \\
80\end{array}$ & $\begin{array}{l}\mathrm{P} \\
\mathrm{N} \\
\mathrm{P} \\
\mathrm{P}\end{array}$ & 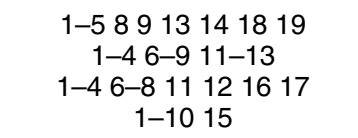 \\
\hline $\begin{array}{l}20.12 .1 \\
20.12 .2 \\
20.12 .3 \\
20.12 .1300\end{array}$ & $\begin{array}{l}(8,212) \\
(8,212) \\
(10,210) \\
(15,205)\end{array}$ & $\begin{array}{l}(72,39,384) \\
(72,39,384) \\
(90,33,372) \\
(135,32,328)\end{array}$ & $\begin{array}{c}(0,12,0,48,48,168,48,204,144,120) \\
(6,6,0,48,36,180,42,210,156,108) \\
(2,8,14,52,72,188,52,150,118,136) \\
(25,34,8,12,70,324,33,152,90,44)\end{array}$ & $\begin{array}{r}1 \\
1 \\
2 \\
51\end{array}$ & $\begin{array}{l}N \\
P \\
N \\
P\end{array}$ & $\begin{array}{c}1-46-911-14 \\
1-3568101113151619 \\
1-681112151618 \\
1-1115\end{array}$ \\
\hline $\begin{array}{l}20.13 .1 \\
20.13 .2 \\
20.13 .3 \\
20.13 .730\end{array}$ & $\begin{array}{l}(14,272) \\
(14,272) \\
(14,272) \\
(18,268)\end{array}$ & $\begin{array}{l}(140,47,528) \\
(140,47,528) \\
(140,47,528) \\
(180,43,492)\end{array}$ & $\begin{array}{l}(0,15,36,96,114,318,72,240,204,192) \\
(7,20,24,80,112,336,72,244,200,192) \\
(10,17,24,72,94,362,62,262,224,160) \\
(39,12,42,84,90,450,48,204,192,126)\end{array}$ & $\begin{array}{r}1 \\
2 \\
1 \\
34\end{array}$ & $\begin{array}{l}N \\
N \\
P \\
P\end{array}$ & $\begin{array}{c}1-46-911-1416 \\
1-911-14 \\
1-35-14 \\
1-710-1215-17\end{array}$ \\
\hline $\begin{array}{l}20.14 .1 \\
20.14 .2 \\
20.14 .3 \\
20.14 .328\end{array}$ & $\begin{array}{l}(20,344) \\
(20,344) \\
(20,344) \\
(22,342)\end{array}$ & $\begin{array}{l}(220,60,721) \\
(220,60,721) \\
(220,60,721) \\
(242,59,700)\end{array}$ & $\begin{array}{l}(0,24,76,170,227,503,125,281,249,347) \\
(0,26,74,166,224,510,125,283,252,342) \\
(0,26,74,166,225,509,126,282,250,344) \\
(49,24,54,108,148,700,74,335,312,198)\end{array}$ & $\begin{array}{r}1 \\
1 \\
1 \\
10\end{array}$ & $\begin{array}{l}Q \\
Q \\
Q\end{array}$ & $\begin{array}{c}1-9 \text { 13 } 14 \text { 16-18 } \\
1-11 \quad 13 \quad 1518 \\
1-1013 \quad 151618 \\
1-8 \quad 10-12 \quad 15-17\end{array}$ \\
\hline
\end{tabular}


Table 5 (continued)

\begin{tabular}{|c|c|c|c|c|c|c|}
\hline Design & $F_{3}$ & $F_{4}$ & $F_{5}$ & GMA & Type & Columns \\
\hline $\begin{array}{l}20.15 .1 \\
20.15 .2 \\
20.15 .3 \\
20.15 .124\end{array}$ & $\begin{array}{l}(26,429) \\
(26,429) \\
(26,429) \\
(27,428)\end{array}$ & $\begin{array}{l}(312,81,972) \\
(312,81,972) \\
(312,81,972) \\
(324,81,960)\end{array}$ & $\begin{array}{c}(0,33,132,264,330,792,165,429,396,462) \\
(0,40,125,262,346,778,181,408,364,499) \\
(0,41,124,260,345,781,180,410,366,496) \\
(65,44,72,148,232,1,040,118,516,464,304)\end{array}$ & $\begin{array}{l}1 \\
1 \\
1 \\
3\end{array}$ & $\begin{array}{l}N \\
Q \\
Q \\
P\end{array}$ & $\begin{array}{c}1-46-911-14 \quad 16-18 \\
1-131718 \\
1-131517 \\
1-12 \quad 15-17\end{array}$ \\
\hline $\begin{array}{l}20.16 .1 \\
20.16 .2 \\
20.16 .3 \\
20.16 .40\end{array}$ & $\begin{array}{l}(32,528) \\
(32,528) \\
(32,528) \\
(33,527)\end{array}$ & $\begin{array}{l}(416,108,1,296) \\
(416,108,1,296) \\
(416,108,1,296) \\
(429,107,1,284)\end{array}$ & $\begin{array}{c}(0,48,192,384,480,1,152,240,624,576,672) \\
(0,60,180,378,504,1,134,264,594,528,726) \\
(14,66,160,332,472,1,212,246,638,564,664) \\
(83,71,104,208,350,1,500,175,747,676,454)\end{array}$ & $\begin{array}{l}1 \\
1 \\
1 \\
2\end{array}$ & $\begin{array}{l}N \\
Q \\
N \\
P\end{array}$ & $\begin{array}{c}1-46-911-14 \quad 16-19 \\
1-131517 \quad 18 \\
1-141617 \\
1-1315-17\end{array}$ \\
\hline $\begin{array}{l}20.17 .1 \\
20.17 .2 \\
20.17 .3 \\
20.17 .11\end{array}$ & $\begin{array}{l}(40,640) \\
(40,640) \\
(40,640) \\
(40,640)\end{array}$ & $\begin{array}{l}(560,140,1,680) \\
(560,140,1,680) \\
(560,140,1,680) \\
(560,140,1,680)\end{array}$ & $\begin{array}{c}(0,91,273,546,728,1,638,364,819,728,1,001) \\
(12,80,272,544,704,1,664,352,832,752,976) \\
(18,94,252,504,692,1,716,346,858,764,944) \\
(108,108,148,296,512,2,104,256,1,052,944,660)\end{array}$ & $\begin{array}{l}1 \\
1 \\
1 \\
1\end{array}$ & $\begin{array}{l}Q \\
N \\
N \\
P\end{array}$ & $\begin{array}{c}1-17 \\
1-911-1416-19 \\
1-1416-18 \\
1-17\end{array}$ \\
\hline $\begin{array}{l}20.18 .1 \\
20.18 .2 \\
20.18 .3 \\
20.18 .6\end{array}$ & $\begin{array}{l}(48,768) \\
(48,768) \\
(48,768) \\
(48,768)\end{array}$ & $\begin{array}{l}(720,180,2,160) \\
(720,180,2,160) \\
(720,180,2,160) \\
(720,180,2,160)\end{array}$ & $\begin{array}{l}(0,126,378,756,1,008,2,268,504,1,134,1,008,1,386) \\
(24,128,352,704,960,2,368,480,1,184,1,056,1,312) \\
(48,144,312,624,912,2,496,456,1,248,1,104,1,224) \\
(140,156,208,416,728,2,888,364,1,444,1,288,936)\end{array}$ & $\begin{array}{l}1 \\
1 \\
1 \\
1\end{array}$ & $\begin{array}{l}Q \\
N \\
N \\
P\end{array}$ & $\begin{array}{c}1-18 \\
1-1416-19 \\
1-18 \\
1-18\end{array}$ \\
\hline $\begin{array}{l}20.19 .1 \\
20.19 .2 \\
20.19 .3\end{array}$ & $\begin{array}{l}(57,912) \\
(57,912) \\
(57,912)\end{array}$ & $\begin{array}{l}(912,228,2,736) \\
(912,228,2,736) \\
(912,228,2,736)\end{array}$ & $\begin{array}{c}(0,171,513,1,026,1,368,3,078,684,1,539,1,368,1,881) \\
(60,192,432,864,1,248,3,360,624,1,680,1,488,1,680) \\
(180,216,288,576,1,008,3,888,504,1,944,1,728,1,296)\end{array}$ & $\begin{array}{l}1 \\
1 \\
1\end{array}$ & $\begin{array}{l}\mathrm{Q} \\
\mathrm{N} \\
\mathrm{P}\end{array}$ & $\begin{array}{l}1-19 \\
1-19 \\
1-19\end{array}$ \\
\hline
\end{tabular}

NOTE: Type $Q$ is a Plackett-Burman design, and types $P$ and $N$ are as described by Deng and Tang (2002).

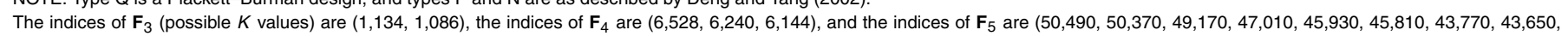
$42,570,42,450)$.

worst projection. This finding partially explains the superiority of type Q, a result also supported by Wang and Wu (1995).

We also study the isomorphism of projection designs from the three Hadamard matrices. Previously, Lin and Draper (1992) studied the isomorphism of projection up to five dimensions, and Wang and Wu (1995) extended it to six dimensions. Deng and Tang (2002) used GMA to rank and classify all projection designs. Table 6 gives the numbers of nonisomorphic designs identified by MAP and GMA. It is evident that MAP identifies many more designs than GMA, as reported by Deng and Tang (2002), especially when the number of columns is large. For example, for 6 columns, GMA identifies only 34 designs whereas MAP identifies 59 designs, which is exactly the number of nonisomorphic designs reported by Wang and $\mathrm{Wu}$ (1995). We point out that Deng and Tang (2002) considered only projection dimensions up to five (or MA-5 in their notation). Nevertheless, the number of nonisomorphic designs found by GMA would be still much less than MAP even if all dimensions are used.

\subsection{Designs of 27 Runs}

Commonly used 27-run designs are regular designs, and minimum aberration is often used to select the best design for a given number of columns (see Wu and Hamada 2000 for details). The 27-run Plackett-Burman design, generated by cyclically permuting the column vector $(0,0,1,0,1,2,1,1,2,0,1$, $1,1,0,0,2,0,2,1,2,2,1,0,2,2,2) 12$ times and adding a row of 0 's, is equivalent to a saturated regular $3^{13-10}$ design. Lam and Tonchev (1996) showed that there are 68 nonisomorphic saturated 27-run OAs with 3 levels and 13 columns, denoted by $O A\left(27,3^{13}\right)$. Among these, only 1 design is regular, and the other 67 designs are nonregular.

Here we use MAP to rank and classify all projection designs from the 68 saturated arrays. For simplicity, we label the $68 O A\left(27,3^{13}\right)$ arrays as types $1-68$ according to their MAP rankings. MAP can separate all but 2 of the 68 arrays; 2 nonisomorphic arrays are ranked as the 24th by MAP. Table 7 lists the top designs and all regular designs identified by MAP. A design is regular if it is from type 68 . Table 7 clearly shows that all regular designs (except for 27.3.1 and 27.4.1) are ranked at the bottom. In other words, according to MAP, nonregular designs are better than regular designs. This agrees with the observation of Cheng and $\mathrm{Wu}$ (2001), who studied the projection properties of three-level designs for a second-order model and concluded that nonregular designs have better projection properties than regular designs.

In Table 7, most of the top designs can be found from type 1 and type 5 arrays, which are given in Table 8 for easy reference. We use the Plackett-Burman design for a type 68 array for convenience.

Table 9 gives the number of nonisomorphic designs identified by MAP and the number of regular designs. Chen, Sun, and Wu (1993) presented a complete catalog of 27-run regular designs with an exhaustive search. It is interesting to note that their catalog is not complete in the sense that they missed a regular design, namely 27.4.139.

Table 6. The Number of 20-Run Nonisomorphic Designs

\begin{tabular}{|c|c|c|c|c|c|c|c|c|c|c|c|c|c|c|c|c|c|}
\hline Method & 3 & 4 & 5 & 6 & 7 & 8 & 9 & 10 & 11 & 12 & 13 & 14 & 15 & 16 & 17 & 18 & 19 \\
\hline MAP & 2 & 3 & 10 & 59 & 388 & 1,265 & 2,089 & 2,282 & 1,899 & 1,300 & 730 & 328 & 124 & 40 & 11 & 6 & 3 \\
\hline GMA & 2 & 3 & 10 & 34 & 51 & 80 & 125 & 125 & 80 & 51 & 34 & 10 & 3 & 2 & 1 & 1 & 1 \\
\hline
\end{tabular}


Table 7. Some 27-Run Designs Ranked by MAP

\begin{tabular}{|c|c|c|c|}
\hline Design & $F_{3}$ & Type & Columns \\
\hline $\begin{array}{l}27.3 .1 \\
27.3 .2 \\
27.3 .3 \\
27.3 .8\end{array}$ & $\begin{array}{l}(0,0,0,0,0,0,0,1) \\
(0,0,0,0,0,0,1,0) \\
(0,0,0,0,0,1,0,0) \\
(1,0,0,0,0,0,0,0)\end{array}$ & $\begin{array}{r}68 \\
5 \\
1 \\
68\end{array}$ & $\begin{aligned} 123 \\
2411 \\
125 \\
126\end{aligned}$ \\
\hline $\begin{array}{l}27.4 .1 \\
27.4 .2 \\
27.4 .3 \\
27.4 .135 \\
27.4 .139\end{array}$ & $\begin{array}{l}(0,0,0,0,0,0,0,4) \\
(0,0,0,0,0,0,2,2) \\
(0,0,0,0,0,0,3,1) \\
(1,0,0,0,0,0,0,3) \\
(4,0,0,0,0,0,0,0)\end{array}$ & $\begin{array}{r}68 \\
5 \\
2 \\
68 \\
68\end{array}$ & $\begin{array}{c}1235 \\
241112 \\
12613 \\
1234 \\
12612\end{array}$ \\
\hline $\begin{array}{l}27.5 .1 \\
27.5 .2 \\
27.5 .3 \\
27.5 .1782 \\
27.5 .1832 \\
27.5 .1833\end{array}$ & $\begin{array}{l}(0,0,0,0,0,3,4,3) \\
(0,0,0,0,0,4,4,2) \\
(0,0,0,0,0,5,2,3) \\
(1,0,0,0,0,0,0,9) \\
(2,0,0,0,0,0,0,8) \\
(4,0,0,0,0,0,0,6)\end{array}$ & $\begin{array}{r}5 \\
8 \\
6 \\
68 \\
68 \\
68\end{array}$ & $\begin{array}{c}4891213 \\
126913 \\
39101213 \\
123410 \\
12345 \\
12348\end{array}$ \\
\hline $\begin{array}{l}27.6 .1 \\
27.6 .2 \\
27.6 .3 \\
27.6 .6191 \\
27.6 .6220 \\
27.6 .6221 \\
27.6 .6230\end{array}$ & $\begin{array}{c}(0,0,0,0,0,14,0,6) \\
(0,0,0,0,2,9,4,5) \\
(0,0,0,0,3,8,4,5) \\
(2,0,0,0,0,0,0,18) \\
(3,0,0,0,0,0,0,17) \\
(4,0,0,0,0,0,0,16) \\
(5,0,0,0,0,0,0,15)\end{array}$ & $\begin{array}{r}37 \\
5 \\
6 \\
68 \\
68 \\
68 \\
68\end{array}$ & $\begin{array}{c}259101213 \\
24891113 \\
569101112 \\
12341011 \\
123457 \\
123456 \\
123458\end{array}$ \\
\hline $\begin{array}{l}27.7 .1 \\
27.7 .2 \\
27.7 .3 \\
27.7 .10284 \\
27.7 .10298 \\
27.7 .10299 \\
27.7 .10300\end{array}$ & $\begin{array}{l}(0,0,0,3,4,15,4,9) \\
(0,0,0,3,6,11,6,9) \\
(0,0,0,4,2,14,6,9) \\
(5,0,0,0,0,0,0,30) \\
(6,0,0,0,0,0,0,29) \\
(7,0,0,0,0,0,0,28) \\
(8,0,0,0,0,0,0,27)\end{array}$ & $\begin{array}{r}5 \\
5 \\
4 \\
68 \\
68 \\
68 \\
68\end{array}$ & $\begin{array}{c}246891113 \\
245891113 \\
23578910 \\
12345710 \\
1234567 \\
1234568 \\
1234589\end{array}$ \\
\hline $\begin{array}{l}27.8 .1 \\
27.8 .2 \\
27.8 .3 \\
27.8 .10269 \\
27.8 .10272 \\
27.8 .10273\end{array}$ & $\begin{array}{c}(0,0,0,6,8,20,8,14) \\
(0,0,0,8,0,31,0,17) \\
(0,0,0,8,0,32,0,16) \\
(8,0,0,0,0,0,0,48) \\
(10,0,0,0,0,0,0,46) \\
(11,0,0,0,0,0,0,45)\end{array}$ & $\begin{array}{r}5 \\
1 \\
1 \\
68 \\
68 \\
68\end{array}$ & $\begin{aligned} 2456891113 \\
1235691011 \\
1235691013 \\
1234571011 \\
12345678 \\
12345689\end{aligned}$ \\
\hline $\begin{array}{l}27.9 .1 \\
27.9 .2 \\
27.9 .3 \\
27.9 .6975 \\
27.9 .6976 \\
27.9 .6977\end{array}$ & $\begin{array}{c}(0,0,0,12,0,48,0,24) \\
(0,0,0,15,0,45,0,24) \\
(0,0,0,15,0,45,0,24) \\
(12,0,0,0,0,0,0,72) \\
(15,0,0,0,0,0,0,69) \\
(16,0,0,0,0,0,0,68)\end{array}$ & $\begin{array}{r}1 \\
1 \\
1 \\
68 \\
68 \\
68\end{array}$ & $\begin{array}{c}123569101113 \\
12345681011 \\
123458101112 \\
123457101112 \\
123456789 \\
1234567812\end{array}$ \\
\hline $\begin{array}{l}27.10 .1 \\
27.10 .2 \\
27.10 .3 \\
27.10 .3304 \\
27.10 .3305\end{array}$ & $\begin{array}{c}(0,0,0,21,0,66,0,33) \\
(0,0,0,21,0,66,0,33) \\
(0,0,0,22,0,65,0,33) \\
(21,0,0,0,0,0,0,99) \\
(22,0,0,0,0,0,0,98)\end{array}$ & $\begin{array}{r}1 \\
1 \\
1 \\
68 \\
68\end{array}$ & $\begin{array}{c}1234567111213 \\
12345678910 \\
123456781011 \\
12345678911 \\
12345678910\end{array}$ \\
\hline $\begin{array}{l}27.11 .1 \\
27.11 .2 \\
27.11 .3 \\
27.11 .1176\end{array}$ & $\begin{array}{c}(0,0,0,30,0,90,0,45) \\
(0,0,0,30,0,90,0,45) \\
(0,0,30,0,30,0,75,30) \\
(30,0,0,0,0,0,0,135)\end{array}$ & $\begin{array}{r}1 \\
1 \\
2 \\
68\end{array}$ & $\begin{array}{l}1234567891113 \\
1234567891011 \\
1234567891011 \\
1234567891011\end{array}$ \\
\hline $\begin{array}{l}27.12 .1 \\
27.12 .2 \\
27.12 .3 \\
27.12 .331\end{array}$ & $\begin{array}{c}(0,0,0,40,0,120,0,60) \\
(0,0,40,0,40,0,100,40) \\
(2,0,0,50,0,84,18,66) \\
(40,0,0,0,0,0,0,180)\end{array}$ & $\begin{array}{r}1 \\
2 \\
3 \\
68\end{array}$ & $\begin{array}{l}123456789101112 \\
123456789101112 \\
123456789101112 \\
123456789101112\end{array}$ \\
\hline $\begin{array}{l}27.13 .1 \\
27.13 .2 \\
27.13 .3 \\
27.13 .67\end{array}$ & $\begin{array}{c}(0,0,0,52,0,156,0,78) \\
(0,0,52,0,52,0,130,52) \\
(3,0,0,63,0,108,27,85) \\
(52,0,0,0,0,0,0,234)\end{array}$ & $\begin{array}{r}1 \\
2 \\
3 \\
68\end{array}$ & $\begin{array}{l}12345678910111213 \\
12345678910111213 \\
12345678910111213 \\
12345678910111213\end{array}$ \\
\hline
\end{tabular}

NOTE: Type 68 is a Plackett-Burman design, and types 1 and 5 are as given in Table 8 .

The indices of $\mathbf{F}_{3}$ (possible $K$ values) are $(972,900,870,864,852,846,834,810$ ).

\section{CONNECTION WITH OTHER DESIGN CRITERIA}

\subsection{Estimation Capacity and Hidden Projection Properties}

Cheng, Steinberg, and Sun (1999) first studied the model robustness of regular minimum aberration designs in terms of estimation capacity. Following their approach, consider models containing all main effects and $f$ two-factor interactions (2fi's for short). Because we do not know in advance which $f$ 2fi's are significant, we consider all possible combinations. Let $E_{f}$ be the number of estimable models and let $D_{f}$ be the average $D$-efficiency of all models, where the $D$-efficiency is 
Table 8. Two 27-Run Saturated Orthogonal Arrays

\begin{tabular}{|c|c|c|c|c|c|c|c|c|c|c|c|c|c|c|c|c|c|c|c|c|c|c|c|c|c|c|c|}
\hline \multicolumn{14}{|c|}{ Type 1} & \multicolumn{14}{|c|}{ Type 5} \\
\hline Run & 1 & 2 & 3 & 4 & 5 & 6 & 7 & 8 & 9 & 10 & 11 & 12 & 13 & Run & 1 & 2 & 3 & 4 & 5 & 6 & 7 & 8 & 9 & 10 & 11 & 12 & 13 \\
\hline 1 & 0 & 0 & 0 & 0 & 1 & 1 & 1 & 1 & 1 & 1 & 1 & 1 & 1 & 1 & 0 & 0 & 0 & 0 & 1 & 1 & 1 & 1 & 1 & 1 & 1 & 1 & 1 \\
\hline 2 & 0 & 0 & 0 & 0 & 2 & 2 & 2 & 2 & 2 & 2 & 2 & 2 & 2 & 2 & 0 & 0 & 0 & 0 & 2 & 2 & 2 & 2 & 2 & 2 & 2 & 2 & 2 \\
\hline 3 & 0 & 2 & 2 & 1 & 0 & 0 & 0 & 1 & 2 & 2 & 2 & 1 & 1 & 3 & 0 & 1 & 1 & 1 & 0 & 0 & 0 & 2 & 2 & 2 & 1 & 1 & 1 \\
\hline 4 & 0 & 2 & 2 & 2 & 2 & 1 & 2 & 0 & 0 & 0 & 1 & 2 & 1 & 4 & 0 & 2 & 2 & 2 & 2 & 2 & 2 & 0 & 0 & 0 & 1 & 1 & 1 \\
\hline 5 & 0 & 1 & 1 & 2 & 0 & 0 & 0 & 2 & 1 & 1 & 1 & 2 & 2 & 5 & 0 & 2 & 2 & 2 & 0 & 0 & 0 & 1 & 1 & 1 & 2 & 2 & 2 \\
\hline 6 & 0 & 1 & 2 & 1 & 1 & 1 & 2 & 2 & 2 & 1 & 0 & 0 & 0 & 6 & 0 & 2 & 2 & 1 & 2 & 1 & 1 & 2 & 2 & 1 & 0 & 0 & 0 \\
\hline 7 & 0 & 2 & 1 & 2 & 2 & 2 & 1 & 1 & 1 & 2 & 0 & 0 & 0 & 7 & 0 & 1 & 1 & 2 & 1 & 2 & 2 & 1 & 1 & 2 & 0 & 0 & 0 \\
\hline 8 & 0 & 1 & 1 & 1 & 1 & 2 & 1 & 0 & 0 & 0 & 2 & 1 & 2 & 8 & 0 & 1 & 1 & 1 & 1 & 1 & 1 & 0 & 0 & 0 & 2 & 2 & 2 \\
\hline 9 & 1 & 0 & 1 & 2 & 0 & 2 & 2 & 0 & 2 & 1 & 0 & 1 & 1 & 9 & 1 & 0 & 1 & 2 & 0 & 1 & 2 & 0 & 2 & 1 & 0 & 2 & 1 \\
\hline 10 & 2 & 0 & 2 & 1 & 0 & 1 & 1 & 0 & 1 & 2 & 0 & 2 & 2 & 10 & 2 & 0 & 2 & 1 & 0 & 2 & 1 & 0 & 1 & 2 & 2 & 0 & 1 \\
\hline 11 & 2 & 0 & 1 & 1 & 2 & 0 & 1 & 2 & 2 & 0 & 1 & 0 & 1 & 11 & 1 & 0 & 2 & 1 & 1 & 0 & 2 & 2 & 1 & 0 & 0 & 1 & 2 \\
\hline 12 & 1 & 0 & 2 & 2 & 1 & 0 & 2 & 1 & 1 & 0 & 2 & 0 & 2 & 12 & 2 & 0 & 1 & 2 & 2 & 0 & 1 & 1 & 2 & 0 & 2 & 1 & 0 \\
\hline 13 & 2 & 0 & 2 & 2 & 1 & 2 & 0 & 2 & 0 & 2 & 1 & 1 & 0 & 13 & 1 & 0 & 1 & 1 & 2 & 2 & 0 & 1 & 0 & 1 & 1 & 0 & 2 \\
\hline 14 & 1 & 0 & 1 & 1 & 2 & 1 & 0 & 1 & 0 & 1 & 2 & 2 & 0 & 14 & 2 & 0 & 2 & 2 & 1 & 1 & 0 & 2 & 0 & 2 & 1 & 2 & 0 \\
\hline 15 & 1 & 1 & 0 & 2 & 0 & 1 & 1 & 2 & 0 & 2 & 2 & 0 & 1 & 15 & 2 & 1 & 0 & 2 & 0 & 2 & 1 & 2 & 0 & 1 & 0 & 1 & 2 \\
\hline 16 & 2 & 2 & 0 & 1 & 0 & 2 & 2 & 1 & 0 & 1 & 1 & 0 & 2 & 16 & 1 & 2 & 0 & 1 & 0 & 1 & 2 & 1 & 0 & 2 & 2 & 1 & 0 \\
\hline 17 & 1 & 1 & 0 & 1 & 2 & 0 & 2 & 0 & 1 & 2 & 1 & 1 & 0 & 17 & 1 & 2 & 0 & 2 & 1 & 0 & 1 & 0 & 2 & 2 & 1 & 0 & 2 \\
\hline 18 & 2 & 2 & 0 & 2 & 1 & 0 & 1 & 0 & 2 & 1 & 2 & 2 & 0 & 18 & 2 & 1 & 0 & 1 & 2 & 0 & 2 & 0 & 1 & 1 & 1 & 2 & 0 \\
\hline 19 & 1 & 2 & 0 & 1 & 1 & 2 & 0 & 2 & 1 & 0 & 0 & 2 & 1 & 19 & 2 & 2 & 0 & 1 & 1 & 2 & 0 & 1 & 2 & 0 & 0 & 2 & 1 \\
\hline 20 & 2 & 1 & 0 & 2 & 2 & 1 & 0 & 1 & 2 & 0 & 0 & 1 & 2 & 20 & 1 & 1 & 0 & 2 & 2 & 1 & 0 & 2 & 1 & 0 & 2 & 0 & 1 \\
\hline 21 & 2 & 2 & 1 & 0 & 0 & 1 & 2 & 2 & 1 & 0 & 2 & 1 & 0 & 21 & 2 & 1 & 2 & 0 & 0 & 1 & 2 & 1 & 2 & 0 & 1 & 0 & 2 \\
\hline 22 & 1 & 1 & 2 & 0 & 0 & 2 & 1 & 1 & 2 & 0 & 1 & 2 & 0 & 22 & 1 & 2 & 1 & 0 & 0 & 2 & 1 & 2 & 1 & 0 & 1 & 2 & 0 \\
\hline 23 & 2 & 1 & 1 & 0 & 1 & 0 & 2 & 1 & 0 & 2 & 0 & 2 & 1 & 23 & 1 & 1 & 2 & 0 & 2 & 0 & 1 & 1 & 0 & 2 & 0 & 2 & 1 \\
\hline 24 & 1 & 2 & 2 & 0 & 2 & 0 & 1 & 2 & 0 & 1 & 0 & 1 & 2 & 24 & 2 & 2 & 1 & 0 & 1 & 0 & 2 & 2 & 0 & 1 & 2 & 0 & 1 \\
\hline 25 & 2 & 1 & 2 & 0 & 2 & 2 & 0 & 0 & 1 & 1 & 2 & 0 & 1 & 25 & 2 & 2 & 1 & 0 & 2 & 1 & 0 & 0 & 1 & 2 & 0 & 1 & 2 \\
\hline 26 & 1 & 2 & 1 & 0 & 1 & 1 & 0 & 0 & 2 & 2 & 1 & 0 & 2 & 26 & 1 & 1 & 2 & 0 & 1 & 2 & 0 & 0 & 2 & 1 & 2 & 1 & 0 \\
\hline 27 & 0 & 0 & 0 & 0 & 0 & 0 & 0 & 0 & 0 & 0 & 0 & 0 & 0 & 27 & 0 & 0 & 0 & 0 & 0 & 0 & 0 & 0 & 0 & 0 & 0 & 0 & 0 \\
\hline
\end{tabular}

calculated as was done by Wang and Wu (1995). One would prefer designs with high estimation capacity $E_{f}$ and large average $D$-efficiency $D_{f}$.

It is known that nonregular designs have some hidden projection properties. Box and Tyssedal (1996) showed that if $N$ is not a multiple of 8 , then any saturated OA with $N$ runs and 2 levels has projectivity 3 ; that is, any three-factor projection contains a complete $2^{3}$ factorial design, possibly with some points replicated. Therefore, any three-factor projection can entertain all three main effects and all 2fi's among them. Cheng (1995) indeed showed that if $N$ is not a multiple of 8 , then any OA with $N$ runs and 2 levels has the following hidden projection property: Any four-factor projection can entertain all 4 main effects and all 2fi's among them. Let $P_{f}$ be the number of $f$-factor projections that can entertain all $f$ main effects and all 2 fi's among them. One would prefer designs with large $P_{f}$ values. Section 4.2 illustrates these concepts in an example.

\subsection{Door Panel Stamping Experiment}

Return to the door panel stamping experiment mentioned in Section 1. The goal is to choose a $20 \times 7$ design with good design properties. Here we evaluate the top 10 and bottom 5 designs given in Table 5 in terms of estimation capacity and hidden projection properties.
First, consider estimation capacity and design efficiency. There are 212 fi's and $\left(\begin{array}{c}21 \\ f\end{array}\right)$ different models with all 7 main effects and $f$ 2fi's. Table 10 gives the results in terms of $D_{f}$ and $N_{f}$ for $f=1, \ldots, 7$, where $N_{f}=\left(\begin{array}{c}21 \\ f\end{array}\right)-E_{f}$ is the number of nonestimable models. All top 10 designs can entertain up to all sets of four 2fi's, whereas all five bottom designs cannot entertain at least nine sets of four 2fi's. All top 10 designs have larger average $D$-efficiency than the bottom 5 designs. It is evident that the top 10 designs are better than the bottom 5 designs in terms of both design efficiency and estimation capacity. Among the top 10 designs, 8 (all except 20.7.4 and 20.7.10) can entertain all sets of $52 \mathrm{fi}$ 's. Their average $D$-efficiencies are pretty close to each other. Note that design 20.7.1, ranked as the first by MAP, is indeed the best in terms of estimation capacity. It has only two nonestimable models with six 2fi's, whereas all other designs have at least five nonestimable models with six 2 fi's.

Next, consider the hidden projection properties of these $20 \times 7$ designs. As discussed earlier, for any OA with 20 runs and 7 columns, any four-factor projection can entertain all 4 main effects and all 2fi's among them. It is interesting to consider five-factor projections here. In Table 10, the last column, $P_{5}$, shows the number of five-factor projections that can entertain all 5 main effects and 10 2fi's among them. Note that the number of estimable models is fairly consistent with the MAP ranking. The top 10 designs can estimate more models

Table 9. The Number of 27-Run Nonisomorphic Designs Identified by MAP

\begin{tabular}{|c|c|c|c|c|c|c|c|c|c|c|c|}
\hline Type & 3 & 4 & 5 & 6 & 7 & 8 & 9 & 10 & 11 & 12 & 13 \\
\hline Total & 8 & 139 & 1,833 & 6,230 & 10,300 & 10,273 & 6,977 & 3,305 & 1,176 & 331 & 67 \\
\hline Regular & 2 & 3 & 3 & 4 & 4 & 3 & 3 & 2 & 1 & 1 & 1 \\
\hline
\end{tabular}


Table 10. Design Efficiency and Estimation Capacity

\begin{tabular}{|c|c|c|c|c|c|c|c|c|c|c|c|c|c|c|c|}
\hline Design & $D_{1}$ & $D_{2}$ & $D_{3}$ & $D_{4}$ & $D_{5}$ & $D_{6}$ & $D_{7}$ & $N_{1}$ & $N_{2}$ & $N_{3}$ & $N_{4}$ & $N_{5}$ & $N_{6}$ & $N_{7}$ & $P_{5}$ \\
\hline 20.7 .1 & .97 & .94 & .92 & .89 & .86 & .82 & .79 & 0 & 0 & 0 & 0 & 0 & 2 & 34 & 21 \\
\hline 20.7 .2 & .97 & .94 & .92 & .89 & .86 & .82 & .79 & 0 & 0 & 0 & 0 & 0 & 6 & 96 & 20 \\
\hline 20.7 .3 & .97 & .94 & .92 & .89 & .86 & .82 & .79 & 0 & 0 & 0 & 0 & 0 & 13 & 197 & 20 \\
\hline 20.7 .4 & .97 & .95 & .92 & .89 & .86 & .83 & .79 & 0 & 0 & 0 & 0 & 1 & 22 & 215 & 20 \\
\hline 20.7 .5 & .97 & .95 & .92 & .89 & .86 & .83 & .79 & 0 & 0 & 0 & 0 & 0 & 6 & 91 & 19 \\
\hline 20.7 .6 & .97 & .95 & .92 & .89 & .86 & .83 & .79 & 0 & 0 & 0 & 0 & 0 & 9 & 138 & 19 \\
\hline 20.7 .7 & .97 & .95 & .92 & .89 & .86 & .83 & .79 & 0 & 0 & 0 & 0 & 0 & 5 & 76 & 18 \\
\hline 20.7 .8 & .97 & .95 & .92 & .89 & .86 & .83 & .79 & 0 & 0 & 0 & 0 & 0 & 5 & 75 & 17 \\
\hline 20.7 .9 & .97 & .94 & .91 & .88 & .85 & .82 & .78 & 0 & 0 & 0 & 0 & 0 & 6 & 93 & 19 \\
\hline 20.7.10 & .97 & .94 & .92 & .89 & .85 & .82 & .78 & 0 & 0 & 0 & 0 & 1 & 22 & 213 & 19 \\
\hline 20.7 .384 & .94 & .88 & .83 & .79 & .74 & .68 & .61 & 0 & 0 & 0 & 9 & 156 & 1,317 & 7,252 & 4 \\
\hline 20.7.385 & .94 & .88 & .84 & .79 & .74 & .68 & .62 & 0 & 0 & 0 & 9 & 153 & 1,264 & 6,798 & 2 \\
\hline 20.7 .386 & .93 & .87 & .82 & .77 & .71 & .65 & .58 & 0 & 0 & 0 & 13 & 231 & 1,986 & 10,825 & 2 \\
\hline 20.7.387 & .93 & .87 & .81 & .76 & .71 & .65 & .57 & 0 & 0 & 0 & 15 & 255 & 2,095 & 10,995 & 0 \\
\hline 20.7 .388 & .91 & .84 & .76 & .68 & .57 & .37 & 0 & 0 & 0 & 0 & 105 & 1,890 & 18,335 & 116,280 & 0 \\
\hline
\end{tabular}

than the bottom 5 designs. Recall that designs 20.7.1, 20.7.2, and 20.7.8 are ranked as the first by GMA. They can estimate 21, 20, and 17 models, which are consistent with their MAP rankings. The top design, 20.7.1, is the only design that can estimate all 21 models; therefore, it is also the best in terms of hidden projection properties.

In conclusion, MAP ranking is supported by estimation capacity and hidden projection properties. For an experiment with 20 runs and 7 factors, such as the door panel stamping experiment, the top design, 20.7.1, is recommended.

\section{CONCLUDING REMARKS}

By an exhaustive computer search, Beder (1998) observed that all 16-run OAs can be embedded in some Hadamard matrices. Therefore, all the 16-run OAs can be found from 5 Hadamard matrices of order 16. However, it is well known that not every OA can be embedded in Hadamard matrices. One can easily find many such examples from 20-run designs. Our goal is to look for new nonisomorphic designs that have good MAP rankings.
For each given number of columns, we used an efficient algorithm due to $\mathrm{Xu}$ (2002) to construct as many as 5,000 OAs. For each OA, we computed its $K$-value distributions and compared them with all $K$-value distributions of projection designs from Hadamard matrices. We found 10, 79, 338, 386, 107, and 15 new OAs for $6,7,8,9,10$, and 11 columns. In particular, we found two new top designs with six columns and two new top designs with seven columns that are not found from the three Hadamard matrices. The 4 new 20-run designs are given in Table 11 . We should mention that a similar effort was done previously by $\mathrm{Li}$ (2000), who searched for new designs by using both GMA and estimation capacity. However, our approach is more straightforward and much easier than his in terms of computation.

Similar to 20-run designs, there are many 27-run OAs that are not part of any saturated $O A\left(27,3^{13}\right)$. In particular, the 27-run OAs with 5-10 columns given by Xu (2002) have less MAP than the top designs given in Table 7.

In conclusion, MAP is a simple, yet powerful tool for ranking and classifying designs. It also provides a nice blueprint to follow when searching for good designs.

Table 11. Top 20-Run Orthogonal Arrays Not From Hadamard Matrices

\begin{tabular}{|c|c|c|c|c|c|c|c|c|c|c|c|c|c|c|c|c|c|c|c|c|c|c|c|c|c|c|c|c|c|}
\hline \multicolumn{7}{|c|}{ New 20.6.1 } & \multicolumn{7}{|c|}{ New 20.6.2 } & \multicolumn{8}{|c|}{ New 20.7.1 } & \multicolumn{8}{|c|}{ New 20.7.2 } \\
\hline Run & 1 & 2 & 3 & 4 & 5 & 6 & Run & 1 & 2 & 3 & 4 & 5 & 6 & Run & 1 & 2 & 3 & 4 & 5 & 6 & 7 & Run & 1 & 2 & 3 & 4 & 5 & 6 & 7 \\
\hline 1 & + & + & + & + & - & - & 1 & + & + & + & - & + & + & 1 & + & + & + & + & + & + & + & 1 & + & + & - & + & - & - & - \\
\hline 2 & + & - & + & - & - & + & 2 & + & - & + & + & + & - & 2 & + & - & + & - & - & + & - & 2 & + & - & - & + & + & + & - \\
\hline 3 & + & + & - & - & + & + & 3 & + & + & - & + & + & - & 3 & + & + & + & - & + & - & - & 3 & + & + & + & + & - & + & - \\
\hline 4 & + & - & - & - & - & - & 4 & + & - & - & - & - & - & 4 & + & - & - & + & - & - & + & 4 & + & - & + & + & - & - & + \\
\hline 5 & + & + & + & + & + & + & 5 & + & + & + & + & - & + & 5 & + & + & + & + & - & - & + & 5 & + & + & - & + & + & + & + \\
\hline 6 & + & - & + & - & + & - & 6 & + & - & - & - & + & + & 6 & + & - & + & + & + & - & - & 6 & + & - & + & - & + & + & + \\
\hline 7 & + & + & - & - & + & - & 7 & + & + & + & - & - & - & 7 & + & + & - & - & + & + & - & 7 & + & + & - & - & - & + & + \\
\hline 8 & + & - & - & + & + & + & 8 & + & - & - & + & + & + & 8 & + & - & - & - & + & + & + & 8 & + & - & + & - & - & - & - \\
\hline 9 & + & + & - & + & - & + & 9 & + & + & - & - & - & + & 9 & + & + & - & - & - & - & + & 9 & + & + & + & - & + & - & + \\
\hline 10 & + & - & + & + & - & - & 10 & + & - & + & + & - & - & 10 & + & - & - & + & - & + & - & 10 & + & - & - & - & + & - & - \\
\hline 11 & - & + & - & + & + & - & 11 & - & + & - & - & + & - & 11 & - & + & - & - & - & - & - & 11 & - & + & + & - & + & + & + \\
\hline 12 & - & - & - & + & - & - & 12 & - & - & + & - & + & - & 12 & - & - & - & + & + & + & - & 12 & - & - & + & + & + & + & - \\
\hline 13 & - & + & + & + & - & + & 13 & - & + & - & + & - & - & 13 & - & + & - & + & - & + & + & 13 & - & + & - & - & - & + & - \\
\hline 14 & - & - & - & + & + & + & 14 & - & - & + & + & - & + & 14 & - & - & + & + & + & - & + & 14 & - & - & - & + & - & + & + \\
\hline 15 & - & + & + & - & - & + & 15 & - & + & + & + & + & - & 15 & - & + & + & + & - & + & - & 15 & - & + & + & + & + & - & - \\
\hline 16 & - & - & - & - & - & + & 16 & - & - & - & - & - & - & 16 & - & - & - & - & + & - & + & 16 & - & - & - & + & + & - & + \\
\hline 17 & - & + & - & - & - & - & 17 & - & + & - & + & + & + & 17 & - & + & + & - & + & + & + & 17 & - & + & - & - & + & - & - \\
\hline 18 & - & - & + & + & + & - & 18 & - & - & + & - & + & + & 18 & - & - & + & - & - & + & + & 18 & - & - & - & - & - & - & + \\
\hline 19 & - & + & + & - & + & - & 19 & - & + & + & - & - & + & 19 & - & + & - & + & + & - & - & 19 & - & + & + & + & - & - & + \\
\hline 20 & - & - & + & - & + & + & 20 & - & - & - & + & - & + & 20 & - & - & + & - & - & - & - & 20 & - & - & + & - & - & + & - \\
\hline
\end{tabular}




\section{ACKNOWLEDGMENTS}

Xu's research was supported by National Science Foundation grant DMS-02-04009. Xu is grateful to Clement Lam for sending the list of 27-run OAs. The authors thank the editor, the associate editor, and the referees for their valuable comments that contributed to the improvement of the article.

[Received July 2002. Revised June 2004.]

\section{REFERENCES}

Beder, J. H. (1998), "Conjectures About Hadamard Matrices," Journal of Statistical Planning and Inference, 72, 7-14.

Booth, K. H. V., and Cox, D. R. (1962), "Some Systematic Supersaturated Designs," Technometrics, 4, 489-495.

Box, G. E. P., and Hunter, J. S. (1961), "The $2^{k-p}$ Fractional Factorial Designs," Technometrics, 3, 311-351, 449-458.

Box, G. E. P., and Tyssedal, J. (1996), "Projective Properties of Certain Orthogonal Arrays," Biometrika, 83, 950-955.

Chen, J., Sun, D. X., and Wu, C. F. J. (1993), "A Catalogue of Two-Level and Three-Level Fractional Factorial Designs With Small Runs," International Statistical Review, 61, 131-145.

Cheng, C. S. (1995), "Some Projection Properties of Orthogonal Arrays," The Annals of Statistics, 23, 1223-1233.

(1998), "Some Hidden Projection Properties of Orthogonal Arrays With Strength Three," Biometrika, 85, 491-495.

Cheng, C. S., Steinberg, D. M., and Sun, D. X. (1999), "Minimum Aberration and Model Robustness for Two-Level Fractional Factorial Designs," Journal of the Royal Statistical Society, Ser. B, 61, 85-93.

Cheng, S.-W., and Wu, C. F. J. (2001), "Factor Screening and Response Surface Exploration" (with discussion), Statistica Sinica, 11, 553-604.

Clark, J. B., and Dean, A. M. (2001), "Equivalence of Fractional Factorial Designs," Statistica Sinica, 11, 537-547.

Dean, A. M., and Draper, N. R. (1999), "Saturated Main-Effect Designs for Factorial Experiments," Statistics and Computing, 9, 179-185.

Deng, L.-Y., and Tang, B. (1999), "Generalized Resolution and Minimum Aberration Criteria for Plackett-Burman and Other Nonregular Factorial Designs," Statistica Sinica, 9, 1071-1082.

(2002), "Design Selection and Classification for Hadamard Matrices Using Generalized Minimum Aberration Criteria," Technometrics, 44, 173-184.

Dey, A., and Mukerjee, R. (1999), Fractional Factorial Plans, New York: Wiley.
Draper, N. R., and Mitchell, T. J. (1968), "Construction of the Set of 256-Run Designs of Resolution $\geq 5$ and the Set of Even 512-Run Designs of Resolution $\geq 6$ With Special Reference to the Unique Saturated Designs," The Annals of Mathematical Statistics, 39, 246-255.

Fries, A., and Hunter, W. G. (1980), "Minimum Aberration $2^{k-p}$ Designs," Technometrics, 22, 601-608.

Hall, M., Jr. (1961), "Hadamard Matrix of Order 16," Jet Propulsion Laboratory Research Summary, 1, 21-26.

(1965), "Hadamard Matrix of Order 20," Jet Propulsion Laboratory Technical Report, 32-761.

Hamada, M., and Wu, C. F. J. (1992), "Analysis of Designed Experiments With Complex Aliasing," Journal of Quality Technology, 24, 130-137.

Hedayat, A. S., Sloane, N. J. A., and Stufken, J. (1999), Orthogonal Arrays: Theory and Applications, New York: Springer-Verlag.

Lam, C., and Tonchev, V. D. (1996), "Classification of Affine-Resolvable 2-(27, 9, 4) Designs," Journal of Statistical Planning and Inference, 56, $187-202$.

Li, Y. (2000), "Constructions of Generalized Minimum Aberration Designs Through Hadamard Matrices and Orthogonal Arrays," unpublished doctoral dissertation, University of Memphis.

Lin, D. K. J., and Draper, N. R. (1992), "Projection Properties of Plackett and Burman Designs," Technometrics, 34, 423-428.

Ma, C.-X., and Fang, K.-T. (2001), "A Note on Generalized Aberration in Factorial Designs," Metrika, 53, 85-93.

Ma, C.-X., Fang, K.-T., and Lin, D. K. J. (2001), "On the Isomorphism of Fractional Factorial Designs," Journal of Complexity, 17, 86-97.

MacWilliams, F. J., and Sloane, N. J. A. (1977), The Theory of Error-Correcting Codes, Amsterdam: North-Holland.

Plackett, R. L., and Burman, J. P. (1946), “The Design of Optimum Multifactorial Experiments," Biometrika, 33, 305-325.

Sun, D. X. (1993), "Estimation Capacity and Related Topics in Experimental Designs," unpublished doctoral thesis, University of Waterloo.

Wang, J. C., and Wu, C. F. J. (1995), "A Hidden Projection Property of PlackettBurman and Related Designs," Statistica Sinica, 5, 235-250.

Wu, C. F. J., and Hamada, M. (2000), Experiments: Planning, Analysis and Parameter Design Optimization, New York: Wiley.

$\mathrm{Xu}$, H. (2002), "An Algorithm for Constructing Orthogonal and NearlyOrthogonal Arrays With Mixed Levels and Small Runs," Technometrics, 44, 356-368.

(2003), "Minimum Moment Aberration for Nonregular Designs and Supersaturated Designs," Statistica Sinica, 13, 691-708.

$\mathrm{Xu}$, H., and Wu, C. F. J. (2001), "Generalized Minimum Aberration for Asymmetrical Fractional Factorial Designs," The Annals of Statistics, 29, 1066-1077.

Yamada, S., and Lin, D. K. J. (1999), "Three-Level Supersaturated Designs," Statistics and Probability Letters, 45, 31-39. 